\title{
Short-term periodicities observed in neutron monitor counting rates
}

\author{
Alejandro López-Comazzi ${ }^{\circledR}$, Juan José Blanco ${ }^{\circledR}$ \\ Correspondence \\ CaLMa - Monitor de Neutrones de Castilla-La Mancha, Universidad de Alcalá, Madrid, Spain, \\ alejandrofrancisco.l@edu.uah.es
}

\section{OPEN ACCESS}

This work is published under th Creative Commons Attribution 4.0 International licence (CC BY 4.0) Please note that individual, appropriately marked parts of the work may be excluded from the licence mentioned or may be subect to mer other copyright conditions. If such thirdparty material is not under the Creative Commons license any copying, editing or public repro duction is only permitted with the prior consent of the respective copyright owner or on the basis of relevant legal authorization regulations.

\section{Keywords}

global neutron monitor; periodicities; solar wind speed; interplanetary magnetic field

\begin{abstract}
The main objective is to check whether the periodicities observed in the cosmic rays in the interval 2013-2018 are affected by the magnetic rigidity or the height at which the neutron monitors are placed. A Global Neutron Monitor (GNM) has been defined as representative of the neutron monitor global network. The Morlet wavelet analysis is applied to the GNM and the selected solar activity parameters to find out common periodicities. Short-term periodicities of $13.5,27,48,92,132$ and 298 days have been observed in cosmic ray intensity. A clear inverse relationship between rigidity and spectral power has been obtained for the $13.5,48,92,132$-day periods. A not so clear but still observed direct relationship between the height of the neutron monitors and the spectral power for the 48,92,132-day periods has been also found. The periodicity of 92 days is the one which shows the highest dependence with rigidity cutoff and height. As far as we know, this is the first time that these dependencies are reported. We think that these observations could be explained by assuming some cosmic ray intensity energy dependence in such periodicities and a competitive effect between rigidity and height.
\end{abstract}

\section{Introduction}

The primary cosmic rays are electrically charged high energy particles, mostly originating in violent phenomena of our galaxy (such as supernova explosions, pulsars with very strong magnetic fields) and, to a lesser extent, solar or extragalactic phenomena, that continuously affect the terrestrial atmosphere with energies between $10^{6}$ and $10^{20} \mathrm{eV} /$ nucleon. When these primary CR interact with particles present in the Earth's atmosphere, other particles called secondary cosmic rays, such as protons, muons, neutrons and mesons are created. Some of these secondary particles are registered by groundbased neutron monitors and muon telescopes respectively.

The year 1964 was designated by cosmic ray investigators as the international quiet year of the sun (IQSY) and Hugh Carmichel developed a neutron monitor (NM) with a statistical accuracy of $0.1 \%$ for hourly data called "NM64" or also known as "supermonitor", Shea and Smart (2000). These "NM64" is used as standard to obtain data which to compare with other stations.

From that date to the present day, other similar instruments have been developed along different locations on the terrestrial sphere. Most of them share their measurements through the Neutron Monitor Data Base (NMDB).

To study the solar activity through NMs requires the use of multiple stations located in different geomagnetic locations because their position with respect to the Earth's magnetic field determines 
the minimum energy of cosmic rays to generate counts on a given NM. This need justifies the foundation of the NMDB.

Cosmic Ray Intensity is anticorrelated to the solar activity, measured by the sunspot number, with a certain delay caused by irregularities in the Interplanetary Magnetic Field, Forbush (1958) and Usoskin et al. (1998).

\section{Results}

\subsection{Data analysis and method}

This proceeding examines the short-term periodicities, i.e. periodicities between 2-512 days, during the period 2013-2018 using the wavelet analysis. The fact that several different phenomena can contribute to different periodicities and these are not constant in time could make Fourier analysis unsuitable (Schreiber et al. 1998) and therefore it has been used wavelet analysis to observe the intrinsic temporal variation in the periods.

A set of neutron monitors with available data from 2013 to 2018 has been selected. These data are one hour pressure corrected counting rates and were collected from the NMDB web page. A check of data availability for the data provider stations in NMDB was performed initially. Only stations with less than $5 \%$ of missing values and/or outliers, i.e. clear wrong data, were selected for the study. The set of outliers and missing values were substituted by linear interpolation. Once the outliers are substituted in the data from the selected stations, the wavelet analysis is applied to their counting rates to find out periodicities in the data. This analysis decomposes a signal into a sum of wavelets which come from a "mother" wavelet function, in this case the Morlet function.

We define as Global neutron monitor (GNM) an ideal neutron monitor whose power spectrum is obtained using the averaged of the counting rates, in typified units, of all the selected neutron monitors in a particular study. Criteria based on three quality indices have been used to define the final list of stations to build the Global neutron monitor. These three quality indices are referenced as $\boldsymbol{Q}_{j}, \boldsymbol{Q}_{\boldsymbol{x}}$ and $\boldsymbol{Q}_{u}$ which, by distinct methods, compare the different stations and give us a quantitative idea of which stations have a distant behavior of the general trend. For the calculation of the averaged counting rates we only took into account the neutron monitors whose value is greater than 0.5 in the three indices.

The index as $\boldsymbol{Q}_{\boldsymbol{j}}$ evaluate how much a single power spectrum of a certain station differs from the one that is obtained by average of all stations. $\boldsymbol{Q}_{\boldsymbol{x}}$ is the weighted cross-correlation between a single neutron monitor counting rates and the other stations. $\boldsymbol{Q}_{\boldsymbol{u}}$ is determined following the same procedure to calculate $\boldsymbol{Q}_{x}$ but it is applied to Global Wavelet Spectrum and not to the counting rates (for more information, see López-Comazzi et al. 2020). The final selection of stations comprises 22 neutron monitors at different vertical cutoff rigidity and height in meters above sea level (m.a.s.l). The positions on the Earth, latitude and longitude, of the selected neutron monitors are depicted in figure 1.

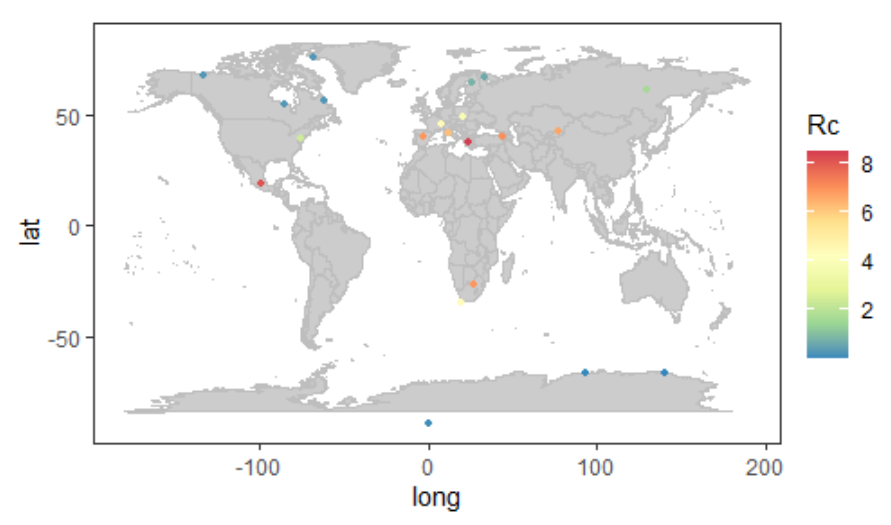

Figure 1: Map with the locations of the 22 selected neutron monitors used to build the Global Neutron Monitor (GNM). 


\subsection{Global Neutron Monitor}

Wavelet analysis applied to the neutron monitor counting rates presents, in general, similar results for all the neutron monitors. The Global Neutron Monitor (GNM) shows a representative behaviour of all stations and it can be used to describe the characteristics of the periodicities observed in cosmic rays counting rates. The detected periodicities in cosmic rays counting rates, total solar irradiance (TSI), sunspot number (SSN), solar wind speed $(V)$ and interplanetary magnetic field $(B)$ are listed in table 1.

The most prominent period observed in the GNM counting rates is the 27-day one and it appears throughout the complete time series except from mid-2013 to mid-2014 (figure 2). This period, related to the synodic solar rotation, was observed by the 22 stations.

A second peak, probably related with solar rotation, is centered in 13.5 days. The 13.5-day period (the second harmonic of the fundamental solar rotation) has been associated with both solar active longitudes as well as tilted dipole structure (Vipindas et al. 2016). This period presents higher power between 2014 and 2017 (figure 2) and it is especially strong in the second half of 2016 during the decreasing phase of the Solar Cycle. This periodicity is much weaker and almost undetectable from 2017 to the end of the studied time interval in coincidence with the solar minimum.

The nearly annual period is the second highest peak (figure 3). Its strength varies with time, being stronger between 2013-2015, weaker between 2015-mid 2017 and disappearing in 2018. This period is clearly observed in all the stations with similar strength. The period is peaked around 298 days.

Three minor peaks (around 132, 92 and 48 days) complete the set of relevant periodicities. All of them show a behavior depending on time disappearing after mid 2015 (figure 2). The three periods were identified between 2014-2016 with similar spectral power in all the neutron monitors and in coincidence with a lower cosmic ray intensity.

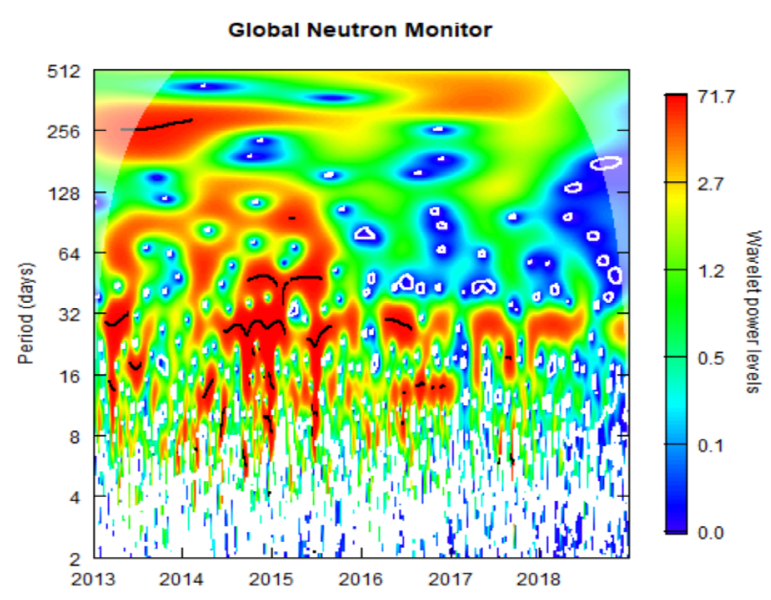

Figure 2: The Wavelet Power Spectrum of the Global Neutron Monitor (GNM). 


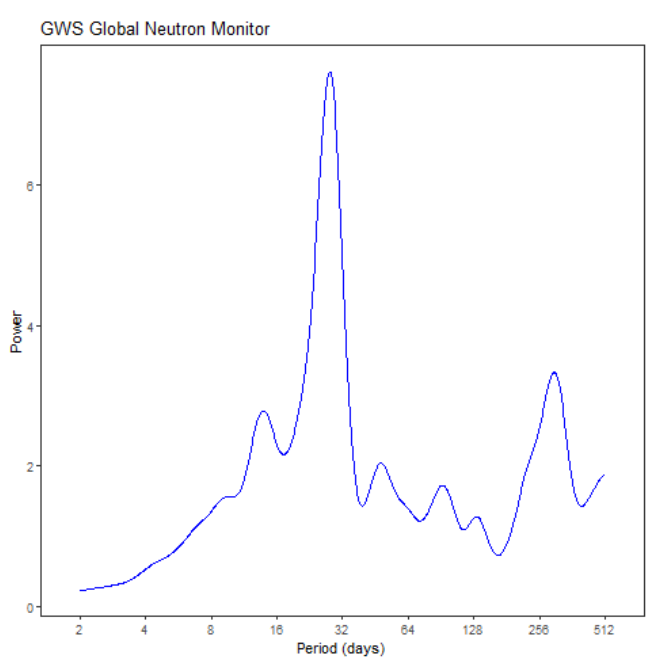

Figure 3: The Global Wavelet Spectrum of the Global Neutron Monitor (GNM).

\begin{tabular}{lrrrrrr}
\hline \multicolumn{7}{c}{ Period (days) } \\
\hline & $\approx 13.5$ & $\approx 27$ & $\approx 48$ & $\approx 92$ & $\approx 132$ & $\approx 298$ \\
\hline$C R$ & $14 \pm 1$ & $28 \pm 3$ & $48 \pm 4$ & $92 \pm 6$ & $132 \pm 5$ & $298 \pm 16$ \\
$V$ & $13 \pm 1$ & $27 \pm 3$ & - & $88 \pm 9$ & - & $256 \pm 28$ \\
$V_{x}$ & $14 \pm 1$ & $27 \pm 3$ & - & $88 \pm 7$ & - & $256 \pm 27$ \\
$V_{\text {perp }}$ & $14 \pm 1$ & $26 \pm 1$ & & $89 \pm 6$ & - & $311 \pm 20$ \\
$B$ & $13 \pm 1$ & $26 \pm 2$ & $54 \pm 3$ & $107 \pm 8$ & - & $298 \pm 29$ \\
$B_{r}$ & $14 \pm 1$ & $27 \pm 3$ & - & - & - & - \\
$B_{z}$ & - & $25 \pm 2$ & - & - & - & - \\
$S S N$ & - & $27 \pm 2$ & $44 \pm 3$ & $105 \pm 7$ & - & $300 \pm 25$ \\
$T S I$ & $14 \pm 1$ & $25 \pm 3$ & $50 \pm 4$ & $94 \pm 5$ & - & $324 \pm 27$ \\
\hline
\end{tabular}

Table 1: Periodicities in GNM counting rates, sunspot number (SSN), total solar irradiance (TSI), interplanetary magnetic field $(B)$ and solar wind speed $(V)$ and its components in GSE coordinates. The second line expresses the notation used to refer to each obtained periodicity in the different ranges.

\subsection{Cutoff rigidity and altitude dependence with spectral power of the different periodicities}

One of the open questions regarding to the observation of periodicities by neutron monitors is the role of rigidity and altitude above sea level on the relative strength of a particular periodicity. The figure 4 shows the spectral power of the 13.5, 48, 92 and 132-day peaks vs magnetic rigidity $\boldsymbol{R}_{c}$. A different behaviour between southern NM (green circles in figure 4) and northern NM (blue circles) has been detected. Southern stations do not show dependence but this is not truth for northern stations.

The dependence of the spectral power with the altitude of the NM site has been also studied. A direct result appears when spectral power is represented against altitude (figure 5). NM at sea level (below 200 m.a.s.l) do not show a relationship with height (green circles in figure 5). However a direct relationship emerges for stations above 200 m.a.s.l (blue circles in figure 5) for the 92 and 132-day periods. 

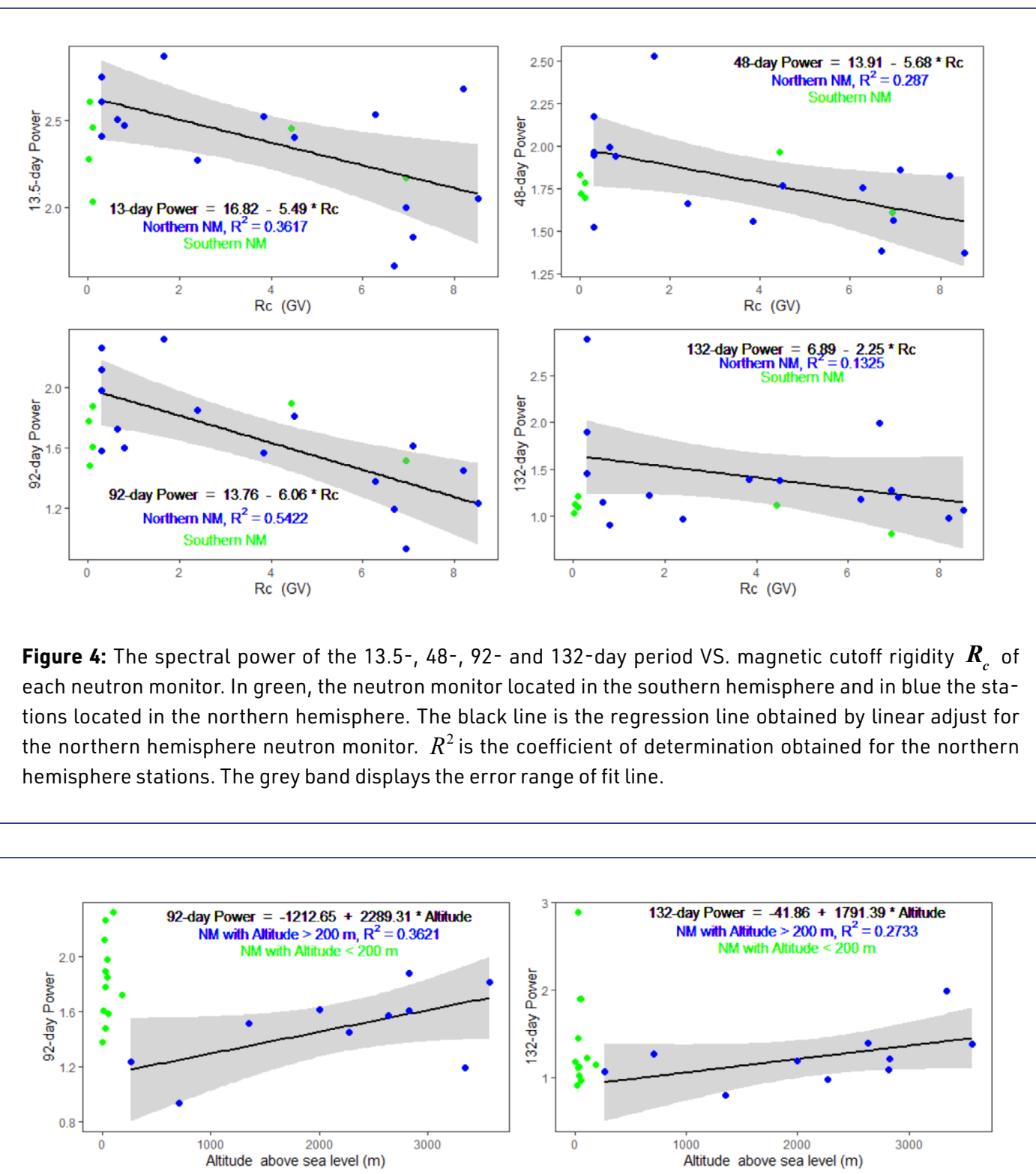

Figure 5: The spectral power of the 92 and 132-day period vs altitude of each neutron monitor in meters above sea level. In green, the neutron monitor with altitude less than $200 \mathrm{~m}$ and in blue the NM with altitude more than $200 \mathrm{~m}$. The black line is the regression line obtained by linear adjust for the NM with altitude higher than $200 \mathrm{~m}$. $R^{2}$ is the coefficient of determination obtained for stations with altitude more than $200 \mathrm{~m}$. The grey band displays the error range of the fit line.

\section{Discussion and conclusions}

A rigidity dependence has been observed in the power of the spectral power in some of the periodicities, concretely in 13.5, 48, 92 and 132-day periods. This result could be explained if the transport conditions are changing with these periods in a way that cosmic rays with less energy are more effective affected than cosmic rays with higher energies.

The second main observation inferred from our work is that also exist a dependence with respect the height at which neutron monitor is located. This dependence is clear at heights above 
$200 \mathrm{~m}$ a.s.l and, as the rigidity dependence, only is observed for certain periodicities. These were the 92 and 132-day periodicities.

The dependence with rigidity and height shows an opposite behaviour regarding to the spectral power on GNM counting rate and both effects are observed for the 92 and 132-day periods. These effects are clearer for the 92-day periodicity.

The periodicities detected in neutron monitor counting rates are also observed in the selected parameters (table 1). We discuss now the periods in parameter and their role in forcing CRI periodicities.

The 48-day period is also observed in the module of interplanetary magnetic field, SSN and TSI while the 92-day period is registered in the same parameters in addition with the solar wind velocity. On the other hand, previous works have reported the relationship between solar wind speed and the fractional coronal hole area (Tulasi et al. 2010) and between the TSI and the presence of coronal holes (Woods et al. 2010). Maybe, this is pointing out a possible relationship between solar wind structures and the observed flux of cosmic ray which is reflected by the observed 48 and 92-day periodicities.

Close to the 92-day period observed in cosmic ray, a periodicity of 88-day has been found in the solar wind velocity. This period is only observed in the solar wind velocity and could be a harmonic of the ACE (spacecraft data used in this work) orbital period of 177 days. Nevertheless after analysing the ACE orbit, no such period of 88 days is detected, therefore it should be concluded that 88 day is a true period in the solar wind velocity. This could reinforce the hypothesis of solar wind structures producing energy depending periodic variations in the cosmic ray flux.

The 13.5 and 27-day periods, related with the solar synodic rotation, are observed in almost all the analyzed parameters. The most prominent peak in the power of the wavelet analysis for NM counting rate is in 27 days (figure 3 ). This period is attributed to the solar rotation commonly, and is related to the coronal holes and co-rotating interaction regions, i.e. interactions between fast and slow solar streams.

The 132-day period was only detected in CR. This could imply that this periodicity is induced by a process not related with solar wind velocity, heliospheric magnetic field, SSN or TSI. However, the relationship of this periodicity with a solar phenomenon cannot be ruled out. It has been reported the observation of the period of 134.5 days in the sunspot data of the northern hemisphere and a similar period (133 day) detected in the data of whole disk along the SC 23 (Joshi et al. 2009).

Finally, the 298-day period is detected in the magnetic field intensity, TSI, SSN and in the component $V_{\text {perp }}$ perpendicular to the ecliptic plane in addition to GNM counting rates.

The 132 and 298-day period are very close to subharmonics of the solar synodic rotation of 27 days, the fifth 135 days and eleventh 298 days) subharmonic. Therefore, the 132 and 298-day period could related to the fifth and eleventh subharmonic of the solar synodic rotation of 27 days.

\section{References}

Forbush, S. E., 1958, J. Geophys. Res., 63, 651, https://ui.adsabs.harvard.edu/abs/1958JGR....63..651F (last accessed April 7, 2021), DOI: https://doi.org/10.1029/JZ063i004p00651

Joshi, B., Pant, P., \& Manoharan, P., 2009, Astronomy and Astrophysics, 452

López-Comazzi, A. Blanco, J.J, 2020, Sol. Phys., 295, 81, DOI: https://doi.org/10.1007/s11207-020-01649-5

Schreiber, H., 1998, Annales Geophysicae, 16, 510, DOI: https://doi.org/10.1007/s00585-998-0510-2

Shea, M. \& Smart, D., 2000, Space Science Reviews, 93, 229, DOI: https://doi.org/10.1023/A:1026500713452

Tulasi Ram, S., Liu, C. H., \& Su, S.-Y., 2010, Journal of Geophysical Research:Space Physics, 115

Usoskin, I. G., Kananen, H., Mursula, K., Tanskanen, P., \& Kovaltsov, G. A., 1998, J. Geophys. Res., 103, 9567, D0I:

https://doi.org/10.1029/97JA03782

Vipindas, V., Gopinath, S., \& Girish, T. E., 2016, Astrophysics and Space Sci-ence, 361, 135. D0I: https://doi. org/10.1007/s10509-016-2719-y

Woods, T. N., 2010, Astronomical Society of the Pacific Conference Series, Vol.428, Irradiance Variations during This Solar Cycle Minimum, ed. S. R. Cranmer, J. T. Hoeksema, \& J. L. Kohl, 63, https://arxiv.org/abs/1003.4524 


\section{Questions and answers}

Monica Laurenza: Are all peaks significant? Did you estimate white or red noise?

Answer: Yes, all peaks are significant. To determine if the peaks represents real characteristics of the time series, a background spectrum is taken. In this work we taken a simple model for red noise, the univariate lag-1 autoregressive process.

Ludwig Klein: The $48 \mathrm{~h}$ period was reported in one of yesterday's talks. Any idea of what it means and if it's real? Answer: I think the 48-day period is real. The 48-day period is observed in this work in neutron monitor counting rates and in the module of interplanetary magnetic field, Sunspot number and total solar irradiance. Previous works have reported the relationship between solar wind speed and the fractional coronal hole area (Tulasi et al. 2010) and between the total solar irradiance and the presence of coronal holes (Woods et al., 2010). Maybe, this is pointing out a possible relationship between solar wind structures and the observed flux of cosmic ray which is reflected by the observed 48-day period.

Ilya Usoskin: Is there correlation between altitude and cutoff rigidity Rc for the analyzed NMs? Most likely highRc NMs tend to be high-altitude.

Answer: This is the plot Altitude VS cutoff rigidity for the selected NMs. Green circles displays southern NMs and blue circles are northern NMs. The linear regression fit give us a coefficient of determination of $R^{2}=0.1$.

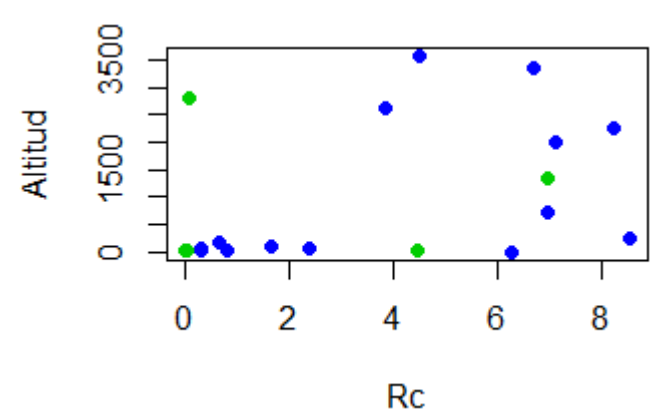

Agnieszka Gil: There is a big difference between sea-level and high-altitude NMs in the 92-day period power vs altitude plot. Any thoughts about the cause? Is the 92-day power vs cutoff rigidity dependence excluded at the plot?

Answer: Probably for stations at sea level or low altitude (less than $200 \mathrm{~m}$ ), the effect of cutoff rigidity is the dominant factor. This fact would explain the strong dispersion of the spectral power value for these stations (NMs with altitude $>200 \mathrm{~m}$ ). In the 92 -day spectral power VS. altitude plot, the 92 -day power vs cutoff rigidity dependency is not excluded.

Gonzalo Tancredi: Do you have plans to extend this work for previous years? e.g. 90's \& 00's

Answer: Yes, I intend to extend the study to the entire range covered by the neutron monitors (1960-2020) and analyze the short- medium- and long-term periodicities. 